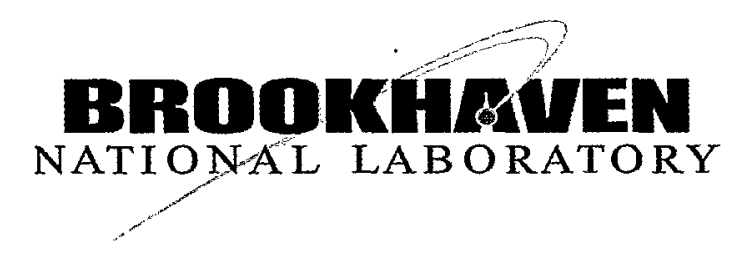

BNL-77496 -2006-CP

\title{
A Study of Polarized Proton Acceleration in J-PARC
}

\author{
A. U. Luccio, M. Bai, T. Roser \\ Brookhaven National Laboratory
}

A. Molodojentsev, C. Ohmori, H. Sato

High Energy Accelerator Research Organization, Tsukuba, Ibaraki, JAPAN

H. Hatanaka

Research Center for Nuclear Physics, Osaka University, JAPAN

Presented at the SPIN 2006 Conference

Kyoto, JAPAN

October 2-7, 2006

November 2006

\author{
Collider-Accelerator Department \\ Brookhaven National Laboratory \\ P.O. Box 5000 \\ Upton, NY 11973-5000 \\ www.bnl.gov
}

\begin{abstract}
Notice: This manuscript has been authored by employees of Brookhaven Science Associates, LLC under Contract No. DE-AC02-98CH10886 with the U.S. Department of Energy. The publisher by accepting the manuscript for publication acknowledges that the United States Government retains a non-exclusive, paid-up, irrevocable, worldwide license to publish or reproduce the published form of this manuscript, or allow others to do so, for United States Government purposes.
\end{abstract}




\section{DISCLAIMER}

This report was prepared as an account of work sponsored by an agency of the United States Government. Neither the United States Government nor any agency thereof, nor any of their employees, nor any of their contractors, subcontractors, or their employees, makes any warranty, express or implied, or assumes any legal liability or responsibility for the accuracy, completeness, or any third party's use or the results of such use of any information, apparatus, product, or process disclosed, or represents that its use would not infringe privately owned rights. Reference herein to any specific commercial product, process, or service by trade name, trademark, manufacturer, or otherwise, does not necessarily constitute or imply its endorsement, recommendation, or favoring by the United States Government or any agency thereof or its contractors or subcontractors. The views and opinions of authors expressed herein do not necessarily state or reflect those of the United States Government or any agency thereof. 


\title{
A Study of Polarized Proton Acceleration in J-PARC ${ }^{1}$
}

\author{
A.U. Luccio, M. Bai, T. Roser, ${ }^{*}$, A. Molodojentsev, C. Ohmori, H. Sato, ${ }^{\dagger}$ \\ and H. Hatanaka** \\ *Brookhaven National Laboratory, Upton, NY 11973, USA \\ †High Energy Accelerator Research Organization, Tsukuba, Ibaraki, Japan \\ ** Research Center for Nuclear Physics, Osaka University, Japan
}

\begin{abstract}
We have studied the feasibility of polarized proton acceleration in rhe J-PARC accelerator facility, consisting of a $400 \mathrm{MeV}$ linac, a $3 \mathrm{GeV}$ rapid cycling synchrotron (RCS) and a $50 \mathrm{GeV}$ synchrotron (MR). We show how the polarization of the beam can be preserved using an $\mathrm{rf}$ dipole in the RCS and two superconductve partial helical Siberian snakes in the MR. The lattice of the MR will be modified with the addition of quadrupoles to compensate for the focusing properties of the snakes.
\end{abstract}

Keywords: polarized protons, acceleration, spin

PACS: $41.75 . \mathrm{Ak}, 07.77 .+\mathrm{p}, 29.27 . \mathrm{Bd}, 29.27 . \mathrm{Hj}$

\section{INTRODUCTION}

The J-PARC faciltity now under construction at the JAEA Tokai site, will focus on accelerating high intensity hadron beams for secondary particle production of neutrons, muons, kaons and neutrinos. With some modifications it would also allow the acceler-

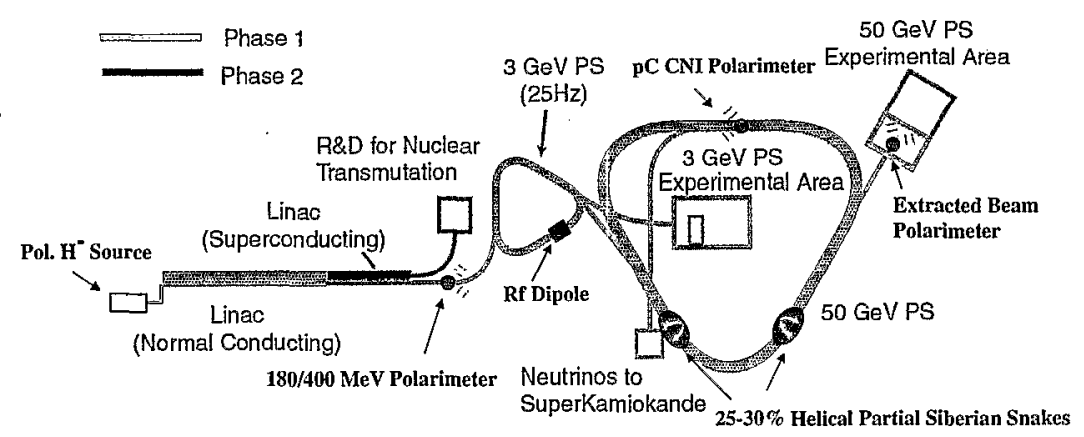

FIGURE 1. Layout of J-PARC accelerator complex and the hardware needed for polarized beam acceleration.

${ }^{1}$ Work performed under the auspices of the US Department of Energy and with support from KEK and Osaka University 
ation of high brightness polarized proton beams to $50 \mathrm{GeV}$ for experiments using this primary beam. The modifications would consist of the addition of a polarized Hminus source, an rf dipole in the $3 \mathrm{GeV}$ Rapid Cycling Synchrotron (RCS) and two strong superconducting partial Siberian snakes in the $50 \mathrm{GeV}$ Main Ring (MR). In addition, several external and internal polarimeters are needed for commissioning and operation of polarized proton acceleration. Fig. 1 shows the layout of J-PARC with the items needed for polarized proton acceleration highlighted. The proposed scheme for the acceleration of polarized protons is based on the successful experience of accelerating polarized protons to $25 \mathrm{GeV}$ at the Brookhaven AGS [1], which is very similar to the J-PARC complex.

The required beam bunch parameters that allow the acceleration of polarized protons to $50 \mathrm{GeV}$ are a normalized $95 \%$ emittance of $10 \pi \mu \mathrm{m}$ and $0.3 \mathrm{eVs}$ longitudinal emittance. With the present avaiable source intensity of $10^{12}$ Hminus for a $0.5 \mathrm{~ms}$ pulse it is easily possible to produce a bunch intensity of $2 \times 10^{11}$ protons for a single bunch in the RCS.

\section{ACCELERATING POLARIZED PROTONS IN THE RCS}

The kinetic energy of the RCS spans from .18 to $3 \mathrm{GeV}(G \gamma=2.2$ to 7.5$)$. The five imperfection resonances can easily be corrected by harmonic orbit correction. With the periodicity 3 and a vertical tune $v_{y}$ of 6.35 there are four intrinsic resonances in this range: $9-v_{y},-3+v_{y}, 12-v_{y}$, and $0+v_{y}$. Simulations showed that the latter three resonances are strong enough so that a modest $20 \mathrm{Gm}$ vertical rf dipole can produce enough coherence to cause complete spin flip. Quite large coherence can be produce in the RCS because of the very large available aperture in this high intensity synchrotron. The first intrinsic resonance is too week for this technique. However, with the fast repetition rate of $25 \mathrm{~Hz}$ the depolarization is only $5 \%$.

\section{ACCELERATING POLARIZED PROTONS IN THE MR}

The main ring [2], designed to accelerate protons from 3 to $50 \mathrm{Gev}(G \gamma=7.5$ to 97.5$)$ at a tune $v_{x}=22.339, v_{y}=20.270$, exhibits many imperfection and strong intrinsic spin resonances, Fig. 2(L). Its lattice is compact and offers no space to insert full Siberian snakes. Available straight sections for snakes are only able to accommodate partial snakes of the type used in the AGS [3].

A solution is obtained by using two $30 \%$ partial snakes separated by $120^{\circ}$ in beam deflection as shown in Fig. 1. Fig. 3, shows the theoretical vertical component of the stable spin axis and the spin tune with two such snakes. The working betatron tunes should be chosen in the two gaps near an integer tune value and the vertical tune needs to be close to an integer that is a multiple of 3 .

Using the tunes 22.128 and 20.960 , Fig. 2(R) shows the results of spin tracking of a beam of 12 particles of emittance $4 \pi \mu \mathrm{m}(1.5 \sigma)$, with two partial snakes.

The J-PARC MR has 3 superperiods, each with an insertion: INSA, INSB and INSC. We have selected two drift sections $3 \mathrm{~m}$ long in INSA and INSB, between the injection 

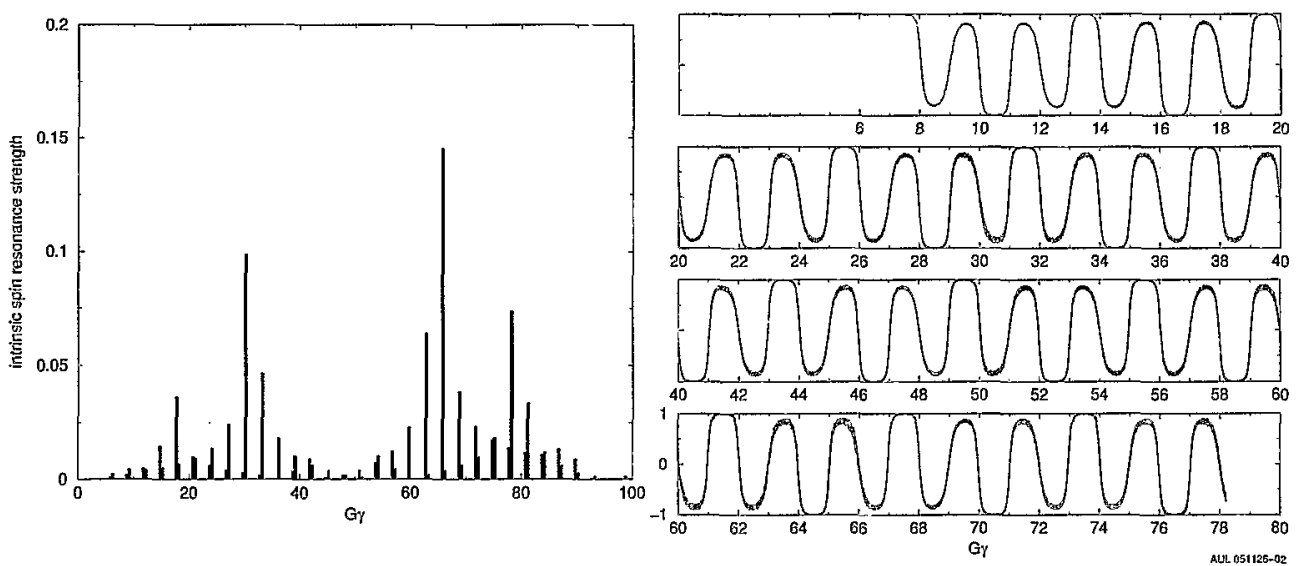

FIGURE 2. (L) Intrinsic spin resonance spectrum for MR. R Spin tracking of a bunch of particles at $1.5 \sigma$ with two $30 \%$ snakes. The average vertical spin $\left\langle S_{y}\right\rangle \pm \sigma$ is shown

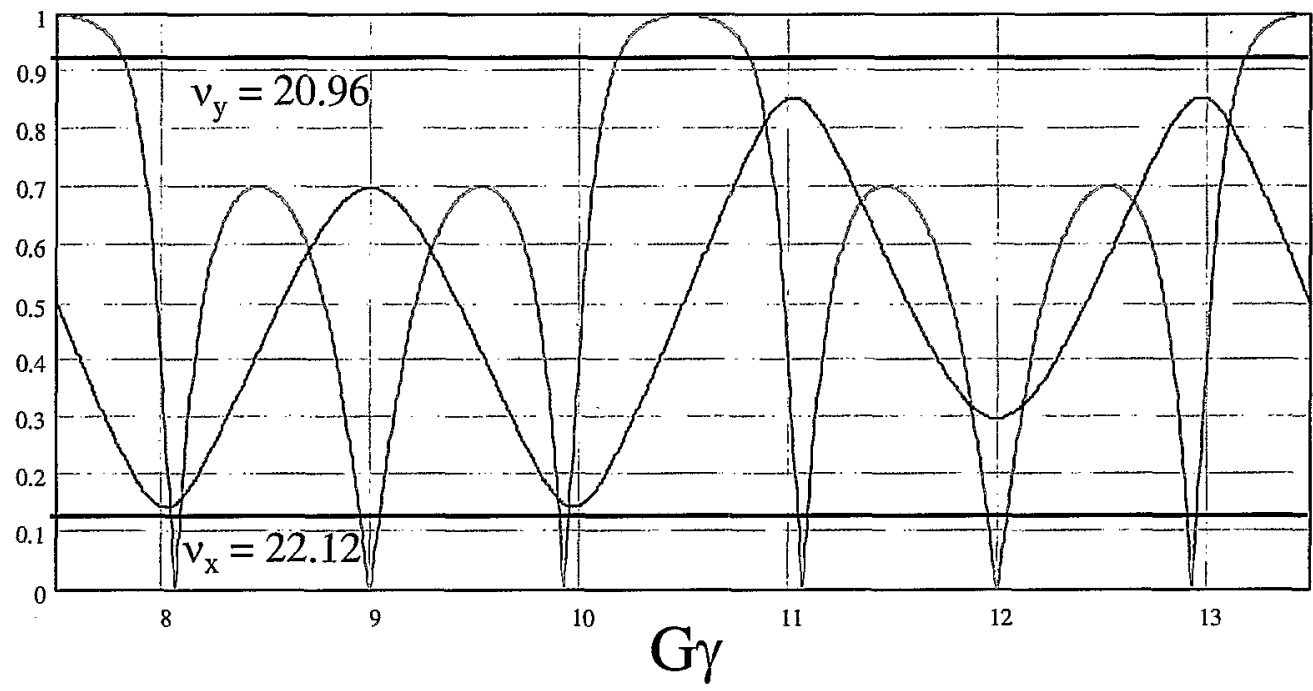

FIGURE 3. Spin tune [blue] and vertical component of the stable spin direction [red] as a fuction of $G \gamma$ for two $30 \%$ partial snakes in the J-PARC MR. $v_{x}$ and $v_{y}$ are placed between the spin tune and the integer.

and slow extraction, where AGS type helical snakes can be placed. Scaling from the AGS, a $30 \%$ snake at injection for the MR could be obtained with a field $B=3.4$ Tesla. The dual pitch helical dipole design of the AGS partial snakes [4] does not affect the beam orbit direction. However, it focuses in both planes. The focusing decreases with beam energy and increases with $B$ as $B^{2} /(\beta \gamma)^{2}$. Because of the strong focusing of the snakes, they produce a substantial perturbation of the lattice at low energies. This can be compensated with two quadrupole doublets on either sides of each snake [6]. We studied a solution using the existing quadrupoles type QDT, QFP, QFT and QFS, that only needs 4 additional power supplies. Required correcting quadrupole strengths decrease 

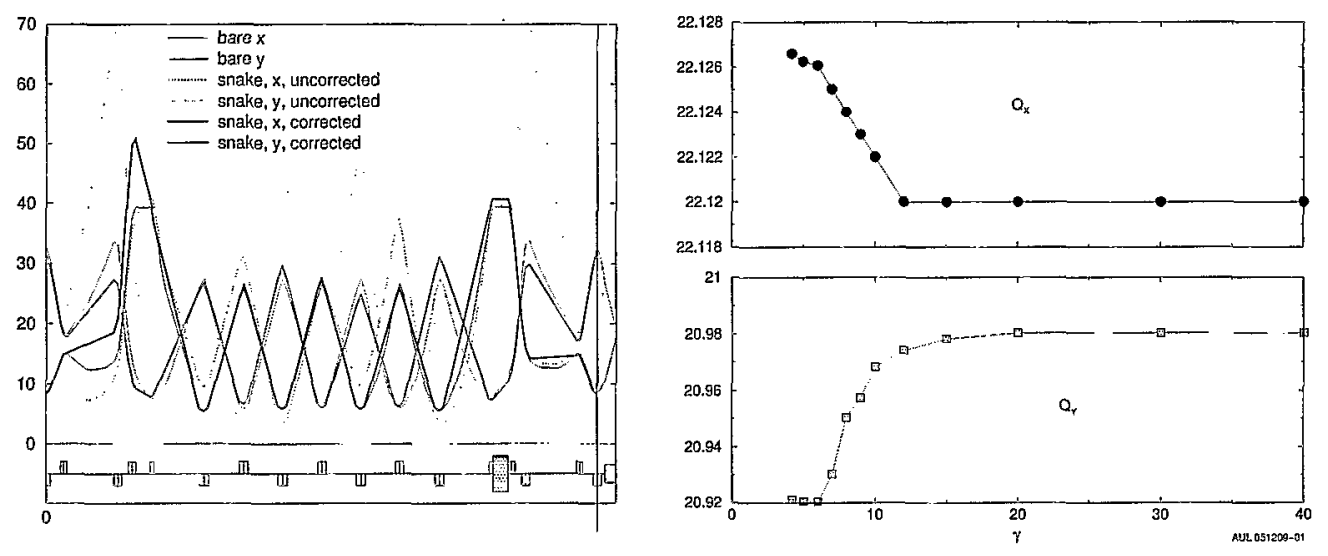

FIGURE 4. (L) Matching of the INSA with snake at the energy $\gamma=11$. (R) Tune path used in the matching of snakes to MR.

as $\left.1 /(\beta \gamma)^{2}\right)$ during acceleration. Fig. $4(\mathrm{~L})$ shows the matching result at $\gamma=11$.

Snake focusing at injection may not allow both horizontal and vertical tunes to be so close to an integer. However, since the spin depolarization resonances in the MR at low energy are very weak, and the amount of depolarization is negligible, we are allowed to ramp the two betatron tunes to near integer starting from values more separated from the integers. A possible tune path is shown in Fig. 4(R).

\section{CONCLUSIONS}

A preliminary study shows that it should be possible to accelerate polarized protons of $10 \pi \mathrm{mm}$-mrad in the J-PARC Main Ring using two $30 \%$ partial snakes of the AGS type.

The perturbation on the MR optics from snakes is larger at low energy. This can be minimized by using correcting quadrupoles at the entrance and exit of each snake. A solution of using two correcting quadrupole doublets per snake is proposed in this paper.

We did tracking with the code Spink using the approximation of synthetic (thin) snakes with variable strength and a static lattice. Further work is needed to do spin tracking with more realistic snake models and with dynamic modeling of the lattice.

\section{REFERENCES}

1. H. Hunag et al., 'Acceleration of polarized protons in the AGS with two helical partial snakes', Proceedings of EPAC2006, Edinburgh, Scotland, p. 273

2. JAERI/KEK JointProjectTeam, Accelerator Technical Design Report for J-PARC, KEK Report 200213, JAERI-Tech 2003-044, J-PARC 03-01, March 2003, A

3. A.U.Luccio,R.Gupta,W.MacKay, and T.Roser, Report C-A/AP/128, December 2003

4. E. Willen et al., 'Superconducting helical snake magnet for the AGS', Proceedings of PAC2005, Knoxville, Tennessee, p. 2935

5. A.U.Luccio, in "Trends in Collider Spin Physics", World Scientific, 1995, p.235

6. E.D.Courant, SPIN2002, AIP Conf. Proc. 675, p.799 